\title{
Local Behavior of the First-Order Gradient Correction to the Thomas-Fermi Kinetic Energy Functional
}

\author{
David García-Aldea $*$ and J. E. Alvarello: $\oplus^{\dagger}$ \\ Departamento de Física Fundamental. UNED. Apartado 60.141. E-28080 Madrid (Spain). \\ T. Martín-Blas \\ Departamento de Ciencias Básicas, E.U.I.T. Forestal, \\ Universidad Politécnica de Madrid. Ciudad Universitaria. E-28040 MADRID (Spain).
}

(Dated: November 20, 2018)

\begin{abstract}
The first order gradient correction to the Thomas-Fermi functional, proposed by Haq, Chattaraj and Deb (Chem. Phys. Lett. 81, 8031, 1984) has been studied by evaluating both the total kinetic energy and the local kinetic energy density. For testing the kinetic energy density we evaluate its deviation from an exact result through a quality factor, a parameter that reflects the quality of the functionals in a better way than their relative errors. The study is performed on two different systems: light atoms (up to $Z=18$ ) and a noninteracting model of fermions confined in a Coulombictype potential. It is found than this approximation gives very low relative errors and a better local behavior than any of the usual generalized gradient approximation semilocal kinetic density functionals.

PACS numbers: 31.15.Ew, 31.15.Bs, 31.15.-p, 71.10.CA, 71.15.Mb
\end{abstract}

\section{INTRODUCTION}

Density Functional Theory, originally developed by Hohenberg and Kohn $(\mathrm{HK}), \frac{1}{\text { r }}$ replaces the main role of the wave function in the description of many electron systems and proves that the particle density $n(\mathbf{r})$ determines all the properties of the system through the functional of the total energy $E[n]$. When $E[n]$ is minimized, the density and the energy of the ground state can be found. The functional for the total energy of the interacting system is unknown, but Kohn and Sham (KS $)^{2}$ devised a method for the minimization of $E[n]$ that uses the concept of noninteracting KS orbitals $\varphi_{i}(\mathbf{r})$ that yield the same density than the interacting system. The KS scheme allows to divide $E[n]$ in four terms, and one of them is the kinetic energy density functional (KEDF) of the KS noninteracting system, $T_{S}[n]$, that can be exactly evaluated in terms of the KS orbitals.

In order to provide insight into the physics of manybody systems, we are interested in elucidating the formal properties of the KEDF. As explained below, the Thomas-Fermi (TF) functional is a local density approximation that has been corrected with other terms that usually depend on the variations of the density, normally including second-order gradient terms (see Ref. 3 for a general review on the results obtained with a number of semilocal kinetic functionals). In this paper we will study the kinetic energy functional that includes the firstorder gradient correction to the TF functional proposed by Haq, Chattaraj and Deb. ity of this first-order gradient correction we will evaluate both the total kinetic energy and the local behavior of the approximate kinetic energy density (KED). The study of the KED (a function that depends on the spatial coordinates) is made through a quality factor $\sigma$, a parameter that reflects the quality of the functionals in a better way than their relative errors $\frac{3,9,10,11,12}{1 t}$ may be expected that the functional which systematically gives the better KED would probably also yield another properties more accurately.

In order to make the study of how this first-order gradient functional works for localized systems we test two simple systems: a noninteracting model (fermions confined in a Coulombic-type potential) and an interacting electron system (light atoms, from He to Ar).

\section{A. Kinetic Energy Functionals}

As commented, the KS scheme allows to define the KEDF of the KS noninteracting system, $T_{S}[n]$, for any many particle system. But, following the original HK idea, many attempts to write the kinetic energy as a functional, using exclusively the electron density, have been made. The very first attempt was a statistical method - the Thomas-Fermi (TF) functional, proposed by Thomas $\frac{13}{13}$ and Fermi $\frac{14}{\underline{1}}$ - that has the form:

$$
T_{T F}[n]=C_{T F} \int d \mathbf{r} n(\mathbf{r})^{5 / 3}, \quad C_{T F}=\frac{3}{10}\left(3 \pi^{2}\right)^{2 / 3} .
$$

$\mathrm{TF}$ is exact for a free electron gas or an uniform system, but this local density approximation yields usually big relative errors -around 10\%- for systems such as atoms and molecules.

Another well-known functional, due to von Weizsäcker $(\mathrm{vW}) \underline{15}$, is exact for any system described by a single orbital,

$$
T_{v W}[n]=\frac{1}{8} \int d \mathbf{r} \frac{|\nabla n(\mathbf{r})|^{2}}{n(\mathbf{r})} .
$$

The TF functional is usually corrected by functionals that depend on the variations of the density, as in 
the second-order Gradient Expansion Approximation) $)^{16}$ (GEA2, where the vW term is weighted by $1 / 9$ )

$$
T_{G E A 2}[n]=C_{T F} \int d \mathbf{r} n(\mathbf{r})^{5 / 3}+\frac{1}{72} \int d \mathbf{r} \frac{|\nabla n(\mathbf{r})|^{2}}{n(\mathbf{r})}
$$

that usually yields small relative errors - usually lower than 1\%- when applied to localized systems using good densities (i. e., those obtained with accurate methods as the Hartree-Fock or the KS ones), although it gives a wrong local behavior, misplacing the KED about $18 \%$. Another functional that has obtained good results for these type of systems (when atomic systems and homonuclear molecules are variationally solved) $)^{17,18}$ is the $\mathrm{TF} \frac{1}{5} \mathrm{vW}$ one,

$$
\begin{aligned}
T_{T F \frac{1}{5} v W}[n] & =T_{T F}[n]+\frac{1}{5} T_{v W}[n] \\
& =C_{T F} \int d \mathbf{r} n(\mathbf{r})^{5 / 3}+\frac{1}{40} \int d \mathbf{r} \frac{|\nabla n(\mathbf{r})|^{2}}{n(\mathbf{r})}(4)
\end{aligned}
$$

But if we use these good electron densities, $\mathrm{TF} \frac{1}{5} \mathrm{vW}$ usually yields bigger relative errors than GEA2 and usually overestimates the total kinetic energy by more than $6 \%$.

Beyond the GEA2 and $\mathrm{TF} \frac{1}{5} \mathrm{vW}$, a number of other semilocal functionals within the Generalized Gradient Approximation (GGA) can be proposed using the general form

$$
T_{G G A}[n]=C_{T F} \int d \mathbf{r} n(\mathbf{r})^{5 / 3} F[s(\mathbf{r})] .
$$

where $F[s(\mathbf{r})]$ is an enhancement factor that corrects the $\mathrm{TF}$ functional via the adimensional reduced gradient,

$$
s(\mathbf{r})=\frac{|\nabla n(\mathbf{r})|}{n(\mathbf{r})^{4 / 3}}
$$

that takes into account the inhomogeneities of the system. The GGA functionals include both the TF functional (with $F[s(\mathbf{r})]=1$ ) and the GEA2 and the $T F \frac{1}{5} \mathrm{vW}$ ones, as well as many other semilocal functionals. The GGA functionals generally depend on $s(\mathbf{r})^{2}$, and their mathematical form and their results are cleary related to the GEA2; only some of the GGAs functionals yield smaller relative errors than the GEA2 but none of them give better KED than the TF functional. ${ }^{3}$

It is also important to know that, due to symmetry considerations in general systems, these functionals depend only on the absolute value of the gradient of the density (or its squared value) and never take into account the directionality of the density variations.

\section{B. First-Order Gradient Correction}

For localized systems a different functional has been proposed by Haq, Chattaraj and Deb (HDC) 4 . This functional is an interesting exception, because it depends on the gradient of the electron density and not on its squared gradient, and also depends on the position vector $\mathbf{r}$ (differently to the other GGAs).

The form of the proposed functional is:

$$
\begin{aligned}
T_{H C D-1}[n(\mathbf{r})] & =C_{T F} \int d \mathbf{r} n(\mathbf{r})^{5 / 3}-\frac{1}{40} \int d \mathbf{r} \frac{\mathbf{r} \nabla n(\mathbf{r})}{r^{2}} \\
& =T_{T F}[n(\mathbf{r})]+T_{r-1}[n(\mathbf{r})],
\end{aligned}
$$

i. e., the sum of the TF functional and an $r$-dependent correction $T_{r-1}[n(\mathbf{r})]$. Note that the $1 / 40$ weight resembles the same prefactor that appeared in the $T F \frac{1}{5} \mathrm{vW}$ functional and that the gradient of the electron density is included in the $r$-dependent term, so this correction is usually labeled as first-order gradient correction (instead of the second-order corrections previously presented).

Making use of the relation

$$
\int d \mathbf{r} \frac{\mathbf{r} \nabla n(\mathbf{r})}{r^{2}}=-\int d \mathbf{r} \frac{n(\mathbf{r})}{r^{2}}
$$

a different first-order term is obtained, and a corresponding functional can be proposed,

$$
T_{r-2}[n(\mathbf{r})]=\frac{1}{40} \int d \mathbf{r} \frac{n(\mathbf{r})}{r^{2}} .
$$

The functional $T_{H C D-2}[n(\mathbf{r})]=T_{T F}[n(\mathbf{r})]+T_{r-2}[n(\mathbf{r})]$ yields the same total kinetic energy but has a different local behavior.

It is also known that $T_{r-1}[n(\mathbf{r})]$ without the $1 / 40$ prefactor is a lower bound ${ }^{19}$ to the Fisher information function, $, 20,21,22 I_{F}[n(\mathbf{r})]$,

$$
-\int d \mathbf{r} \frac{\mathbf{r} \nabla n(\mathbf{r})}{r^{2}} \leq \int d \mathbf{r} \frac{|\nabla n(\mathbf{r})|^{2}}{n(\mathbf{r})}=I_{F}[n(\mathbf{r})] .
$$

On the other hand, the Fisher information function is directly related related to the vW functional,

$$
T_{v W}[n]=\frac{1}{8} I_{F}[n(\mathbf{r})]
$$

so $\frac{1}{5} T_{v W}[n]$ is an upper bound to $T_{r-1}[n(\mathbf{r})]$ and $T_{T F \frac{1}{5} v W}[n(\mathbf{r})]$ becomes an upper bound to the HCD functional,

$$
T_{H C D}[n(\mathbf{r})] \leq T_{T F \frac{1}{5} v W}[n(\mathbf{r})] .
$$

For all these results, the functional $T_{H C D}[n(\mathbf{r})]$ could be a promising functional yielding small relative errors.

Note that $T_{r-2}[n(\mathbf{r})]$ is the average of $r^{-2}$ over the system, $\left\langle r^{-2}\right\rangle=\int d \mathbf{r} \frac{n(\mathbf{r})}{r^{2}}$, with a $1 / 40$ prefactor. This quantity is related to exact bounds for the von Weizsäcker functional, being particularly interesting the following one:

$$
\frac{1}{8}\left\langle r^{-2}\right\rangle<T_{v W}[n(\mathbf{r})]<\frac{1}{4}\left\langle r^{-2}\right\rangle .
$$

The lower bound is of variational origin while the upper bound is based on the complete monotonicity hypothesis. 
(see a general discussion on this topic in Ref. (23) and references therein).

We must note that the choice of $1 / 40$ as a prefactor has no theoretical justification and was chosen by trial and error. But it is possible to make a qualitative exercise to make a guess of the approximate value of this prefactor. We can argue using the previous inequality that the kinetic energy obtained with the vW functional should be close to the mean of the two bounds, i.e.

$$
\frac{3}{16}\left\langle r^{-2}\right\rangle \approx T_{v W}[n(\mathbf{r})]
$$

On the other hand, it is well known that the TF functional can be quite accurately corrected by adding a weighted $\mathrm{vW}$ term (e.g. the GEA2 functional usually yields errors lower than 1\%). And by replacing the $\mathrm{vW}$ functional by $\frac{3}{16}\left\langle r^{-2}\right\rangle$, we add to the TF functional a correction that is $\frac{1}{48}\left\langle r^{-2}\right\rangle$. This value $1 / 48$ is quite close to the proposed $1 / 40$ (a difference of $17 \%$, for a correction that represents about $8 \%$ of the total kinetic energy).

\section{The Relative Errors and the Local Kinetic Behavior}

As pointed out before, the present paper is devoted to the study of the two functionals proposed in Sect. (IB), $T_{H C D}[n(\mathbf{r})]$, with an analysis of both their total kinetic energy and their KED. We study two different systems with spherical symmetric densities. Firstly, we use the electron densities of light atoms from $Z=2$ to $Z=18$. As a second system, we will study the noninteracting fermion system confined in a Coulomb-type potential presented in Ref. (12). In both cases, we have worked with the analytical expressions of the single-particle orbitals in order to evaluate not only the relative errors obtained by the approximate KEDFs, but also compare their KED to the exact $\mathrm{KS}$ results (that can be directly obtained from these orbitals). To make that comparison we have introduced $3,9,10,11,12$ a quantity $\sigma$ that takes into account the local differences, focuses on the error cancellations, and reflects the fact that the KED is not univocally defined.

Let's do a brief review of the method. For a given set of $N$ orbitals $\varphi_{i}(\mathbf{r})$ we can use the usual orbital-based KED, $t_{S}^{I}(\mathbf{r})=\frac{1}{2} \sum_{i=1}^{N}\left|\nabla \varphi_{i}(\mathbf{r})\right|^{2}$, or another common definition of the KED, $t_{S}^{I I}(\mathbf{r})=-\frac{1}{2} \sum_{i=1}^{N} \varphi_{i}^{*}(\mathbf{r}) \nabla^{2} \varphi_{i}(\mathbf{r})$. But for this set of $N$ orbitals we can construct an infinite number of valid KEDs using a linear combination of $t_{S}^{I}$ and $t_{S}^{I I}$,

$$
\begin{aligned}
t_{S}^{L, \alpha}(\mathbf{r})= & (1-\alpha) t_{S}^{I}(\mathbf{r})+\alpha t_{S}^{I I}(\mathbf{r}) \\
= & (1-\alpha) \frac{1}{2} \sum_{i=1}^{N}\left|\nabla \varphi_{i}(\mathbf{r})\right|^{2} \\
& +\alpha \sum_{i=1}^{N}\left(-\frac{1}{2} \varphi_{i}^{*}(\mathbf{r}) \nabla^{2} \varphi_{i}(\mathbf{r})\right) \\
= & t_{S}^{I}(\mathbf{r})-\frac{1}{4} \alpha \nabla^{2} n(\mathbf{r})
\end{aligned}
$$

(all of them are valid KED, see Ref. (24)). So, $t_{S}^{L, \alpha}(\mathbf{r})$ has a first term $t_{S}^{I}(\mathbf{r})$ given by the orbitals plus a term proportional to the laplacian of the electron density (the laplacian integrates to zero in the whole space). The real parameter $\alpha$ can be arbitrarily varied and the family of KEDs described by $t_{S}^{L, \alpha}(\mathbf{r})$ includes $t_{S}^{I}(\mathbf{r})$ (when $\alpha=0$ ) and $t_{S}^{I I}(\mathbf{r})($ for $\alpha=1)$.

When an approximate KEFD is used,each value of $\alpha$ defines a quality factor

$$
\sigma(\alpha)=\frac{\int d \mathbf{r}\left|t_{S}^{L, \alpha}(\mathbf{r})-t_{S}^{f u n c}(\mathbf{r})\right|}{T_{S}[n]},
$$

being $t_{S}^{f u n c}(\mathbf{r})$ the approximate KED of the kinetic functional. $\sigma$ represents a summation of the absolute value of the differences between KEDs, so it measures how the approximated KED is misplaced with respect to the distribution $t_{S}^{L, \alpha}(\mathbf{r})$. Note that $\sigma$ is zero if the approximate and exact distributions of KED are identical, and when the larger the value of $\sigma$ is the bigger the differences between the two are. For each approximate functional we will obtain a set of values of $\sigma(\alpha)$ and in order to compare the KEDs we choose the best value of $\alpha$ for each functional, i. e. the value of $\alpha_{\min }$ that yields the minimum value of $\sigma(\alpha)$; the KED has its closest $t_{S}^{L}(\mathbf{r})$ by evaluating Eq. (12) for this value $\alpha_{\min }$. For details on this measure $\sigma$, the behavior of GGA functionals and the non uniqueness in the definition of the exact KED, see Ref. (3) and references therein.

\section{RESULTS AND DISCUSSION}

In order to assess the quality of the two HCD, $r$ dependent functionals, introduced in Sec. IB (HCD-1 and HCD-2), we are going to compare both the relative errors in the kinetic energies and the quality factor $\sigma$ of the KED obtained with these functionals to the values of the same magnitudes obtained with the TF, GEA2 and $\mathrm{TF} \frac{1}{5} \mathrm{vW}$ functionals.

We have applied the functionals to two different systems. Firstly we study the atoms from He to Ar (from $Z=2$ to $Z=18$ ). We will approximate the atomic orbitals by orthogonalized Slater orbitals, because they do not add any spurious oscillations in the laplacian of 


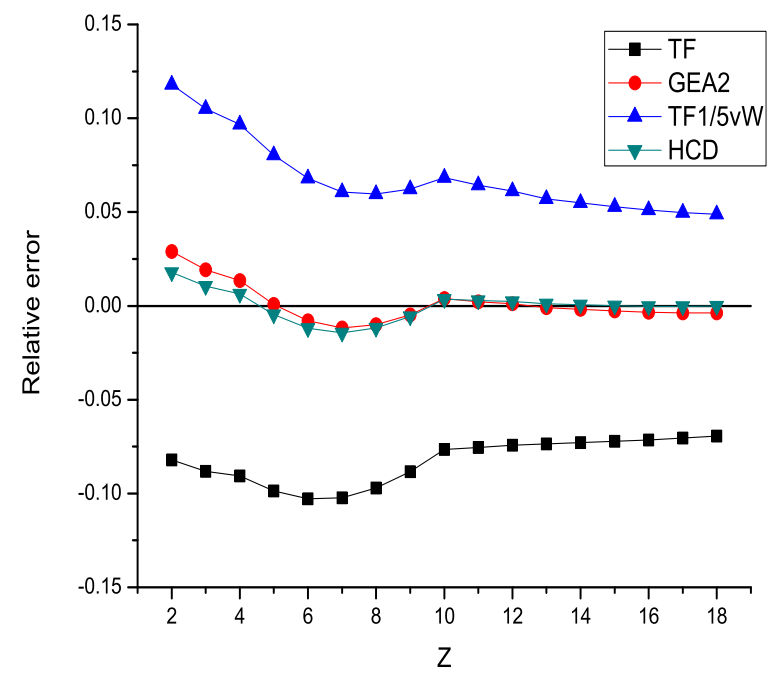

FIG. 1: (Color online) Relative errors of the TF, GEA2, $\mathrm{TF} \frac{1}{5} \mathrm{vW}$ and the first order correction HCD functionals for atoms from $\mathrm{He}$ to Ar.

the density and both the correct cusp behavior and the density decay for $r \rightarrow \infty$ can be achieved (the Gaussiantype basis sets have well known deficiencies, they cannot reproduce the cusps of electronic density and electronic orbitals on nuclei and cannot provide the correct asymptotic behavior at long distances from the nuclei). Being the orbitals known, the local behavior of the KED can be exactly studied. The values of the exponents for the Slater orbitals 25,26 and the corresponding exact total kinetic energy for the atoms used in this paper are shown in Table I]

Fig. 1 shows the values of the relative errors of the total kinetic energy yielded by the functionals for the atoms, as a function of the atomic number $Z$. The best results are obtained for the GEA2 and the two HCD functionals, with quite close errors. The TF functional gives a negative relative error, whereas the $\mathrm{TF} \frac{1}{5} \mathrm{vW}$ shows a similar trend, but with positive errors and smaller absolute values. In all cases, the relative errors for all theses KEDFs are almost constant for $Z \geq 10$.

For the atoms, the value of $\sigma$ is smaller the bigger $Z$ is, as shown in Fig. 2 where the values of the quality factor are plotted as a function of $Z$. The results clearly show that the HCD-2 functional give the smallest values of $\sigma$, reflecting the better local behavior of this semilocal functional. We have found that the GGA corrections to the TF functional improve the TF results for the total kinetic energies, but they always give worse local KEDs, an unexpected failure that shows that GGA functionals are unable to improve the local pathologies of the TF functional.$\underline{3}$ This failure is overcome by the HCD-2 form of the first-order correction, whereas the HCD-1 form

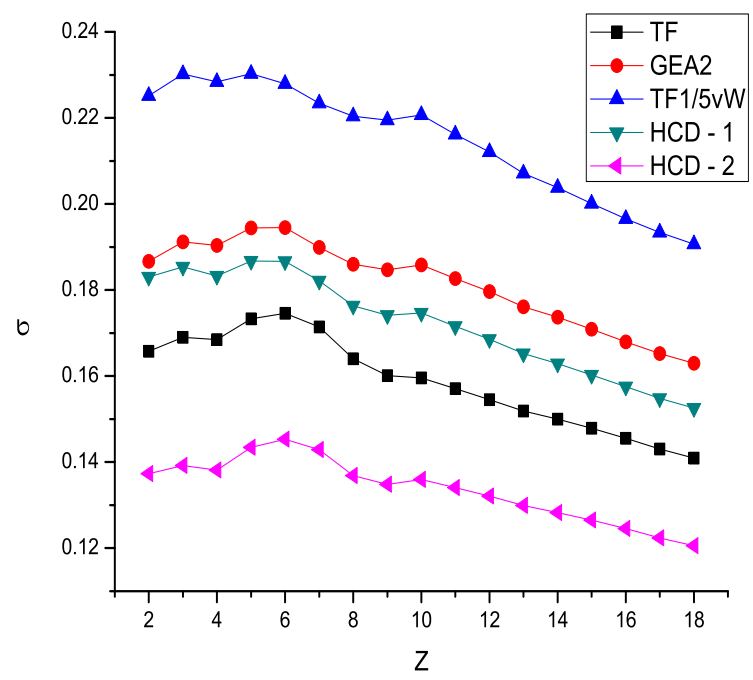

FIG. 2: (Color online) Quality factors $\sigma$ obtained with the $\mathrm{TF}, \mathrm{GEA} 2, \mathrm{TF} \frac{1}{5} \mathrm{vW}$ and the first order correction HCD functionals for atoms from He to Ar.

improves the total kinetic energies, but not the value of $\sigma$, as can be seen in Figs. 1 and 2,

The second test system is constructed with a set of noninteracting fermions that are confined in a Coulombic potential $\stackrel{12}{12}$ In this case, we can also use analytical single-particle orbitals, those of a Coulombic-type potential. We will use spherically symmetric densities for this $N$-fermion system built up with mixed states and we will describe the degenerate $N$-fermion states that arise from the distinct values of the occupancy of the $l$ single-fermion states. These degenerate $N$-fermion states have a different orbital angular momentum for each configuration, with a corresponding different density $n(\mathbf{r}) \stackrel{12}{=}$ As the HK theorems hold not only for densities that correspond to a ground-state density for some external potential ${ }^{\underline{1}}$ but also for a weighted average of ground-state electron densities corresponding to that potential, $, 27,28,29,30$ we can directly discuss the densities corresponding to a degenerate ground-state $\underline{30,31,32,33}$

For the sake of simplicity we will only present the detailed results for the noninteracting systems with a number of fermions $N$ between 1 and 10. In Table【the occupation numbers of each of the subshells of single-fermion orbitals $1 s, 2 s$ and $2 p$, and the value of $\sum_{i=1}^{N} \sqrt{l_{i}\left(l_{i}+1\right)}$ for each of the $N$-fermions configuration are shown. As commented in Ref. (12), $\sum_{i=1}^{N} \sqrt{l_{i}\left(l_{i}+1\right)}$ can be intuitively related to an angular momentum of the configuration, although it is not the expected value of the angular momentum operator but the sum of the eigenvalues of $\sqrt{L^{2}}$ for each independent single-fermion orbital. In the table we also show the relative errors $\epsilon$ obtained with the KEDFs and the values of $\sigma_{\min }$ that give the closest 
TABLE I: Values of the Slater orbital exponents and the corresponding exact total kinetic energy for the atoms studied in the paper.

\begin{tabular}{c|ccccccccc}
\hline \hline Atom & $\mathrm{He}$ & $\mathrm{Li}$ & $\mathrm{Be}$ & $\mathrm{B}$ & $\mathrm{C}$ & $\mathrm{N}$ & $\mathrm{O}$ & $\mathrm{F}$ & $\mathrm{Ne}$ \\
\hline $1 \mathrm{~s}$ & 1.6875 & 2.6906 & 3.6848 & 4.6795 & 5.6727 & 6.6651 & 7.6579 & 8.6501 & 9.6421 \\
$2 \mathrm{~s}$ & & 0.6396 & 0.9560 & 1.2881 & 1.6083 & 1.9237 & 2.2458 & 2.5638 & 2.8792 \\
$2 \mathrm{p}$ & & & & 1.2107 & 1.5679 & 1.9170 & 2.2266 & 2.5500 & 2.8792 \\
\hline Exact KE & 2.8477 & 7.4421 & 14.556 & 24.498 & 37.620 & 54.281 & 74.538 & 98.996 & 127.80 \\
\hline \hline Atom & & $\mathrm{Na}$ & $\mathrm{Mg}$ & $\mathrm{Al}$ & $\mathrm{Si}$ & $\mathrm{P}$ & $\mathrm{S}$ & $\mathrm{Cl}$ & $\mathrm{Ar}$ \\
\hline $1 \mathrm{~s}$ & 10.6259 & 11.6089 & 12.591 & 13.5745 & 14.5578 & 15.5409 & 16.5239 & 17.5075 \\
$2 \mathrm{~s}$ & 3.2857 & 3.6960 & 4.1068 & 4.5100 & 4.9125 & 5.3144 & 5.7152 & 6.1152 \\
$2 \mathrm{p}$ & 3.4009 & 3.9129 & 4.4817 & 4.9725 & 5.4806 & 5.9885 & 6.4966 & 7.0041 \\
$3 \mathrm{~s}$ & 0.8358 & 1.1025 & 1.3724 & 1.6344 & 1.8806 & 2.1223 & 2.3561 & 2.5856 \\
$3 \mathrm{p}$ & & & & 1.3552 & 1.4284 & 1.6288 & 1.8273 & 2.0387 & 2.2547 \\
\hline Exact KE & 161.14 & 198.82 & 242.86 & 289,19 & 340.88 & 397.34 & 458.88 & 525.59 \\
\hline \hline
\end{tabular}

TABLE II: Relative errors $\epsilon$ and optimized values of the quality factor $\sigma_{m i n}$ for all the configurations of the noninteracting $N$-fermion system from $N=1$ to 10 . The occupation numbers $p$ of each orbital is shown, as well as the values of $\sum_{i=1}^{N} l_{i}\left(l_{i}+1\right)$.

\begin{tabular}{|c|c|c|c|c|c|c|c|c|c|c|c|c|c|c|}
\hline & \multicolumn{4}{|c|}{ Orbital occupation } & \multicolumn{2}{|c|}{ TF } & \multicolumn{2}{|c|}{$\mathrm{TF} \frac{1}{5} \mathrm{vW}$} & \multicolumn{2}{|c|}{ GEA2 } & \multicolumn{2}{|c|}{ HCD-1 } & \multicolumn{2}{|c|}{ HCD-2 } \\
\hline$N$ & $p_{1 s}$ & $p_{2 s}$ & $p_{2 p}$ & $\sum_{i=1}^{N} l_{i}\left(l_{i}+1\right)$ & $\sigma_{\min }$ & $\epsilon$ & $\sigma_{\min }$ & $\epsilon$ & $\sigma_{\min }$ & $\epsilon$ & $\sigma_{\min }$ & $\epsilon$ & $\sigma_{\min }$ & $\epsilon$ \\
\hline 1 & 1 & 0 & 0 & 0 & 0.422 & -0.422 & 0.222 & -0.222 & 0.311 & -0.311 & 0.322 & -0.322 & 0.330 & -0.322 \\
\hline 2 & 2 & 0 & 0 & $\overline{0}$ & 0.166 & -0.082 & 0.225 & 0.118 & 0.187 & $\begin{array}{l}0.029 \\
\end{array}$ & \begin{tabular}{|l|l|}
0.171 \\
\end{tabular} & 0.018 & 0.137 & 0.018 \\
\hline 3 & 2 & 1 & 0 & 0 & 0.179 & -0.107 & 0.236 & 0.073 & 0.200 & -0.007 & 0.182 & -0.013 & 0.148 & -0.013 \\
\hline 3 & 2 & 0 & 1 & 2 & 0.198 & -0.126 & 0.244 & 0.041 & 0.215 & -0.033 & 0.201 & -0.036 & 0.170 & -0.036 \\
\hline 4 & 2 & 2 & 0 & 0 & 0.180 & -0.114 & 0.233 & 0.057 & 0.199 & -0.019 & 0.182 & -0.024 & 0.149 & -0.024 \\
\hline 4 & 2 & 1 & 1 & 2 & 0.196 & -0.132 & 0.238 & 0.026 & 0.211 & -0.044 & 0.197 & -0.046 & 0.166 & -0.046 \\
\hline 4 & 2 & 0 & 2 & 4 & 0.209 & -0.145 & 0.241 & 0.003 & 0.220 & -0.062 & 0.210 & -0.061 & 0.182 & -0.061 \\
\hline 5 & 2 & 2 & 1 & 2 & 0.187 & -0.127 & 0.227 & 0.026 & 0.201 & -0.042 & 0.187 & -0.043 & 0.158 & -0.043 \\
\hline 5 & 2 & 1 & 2 & 4 & 0.197 & -0.139 & 0.228 & 0.003 & 0.207 & -0.060 & 0.197 & -0.059 & 0.169 & -0.059 \\
\hline 5 & 2 & 0 & 3 & 6 & 0.206 & -0.147 & 0.227 & -0.013 & 0.212 & -0.073 & 0.205 & -0.070 & 0.180 & -0.070 \\
\hline 6 & 2 & 2 & 2 & 4 & 0.182 & -0.127 & 0.213 & 0.012 & 0.192 & -0.049 & 0.181 & -0.049 & 0.155 & -0.049 \\
\hline 6 & 2 & 1 & 3 & 6 & 0.188 & -0.135 & 0.209 & -0.004 & 0.194 & -0.062 & 0.187 & -0.060 & 0.161 & -0.060 \\
\hline 6 & 2 & 0 & 4 & 8 & 0.193 & -0.139 & 0.206 & -0.016 & 0.195 & -0.070 & 0.191 & -0.067 & 0.168 & -0.067 \\
\hline 7 & 2 & 2 & 3 & 6 & 0.169 & -0.117 & 0.193 & 0.011 & 0.176 & -0.046 & 0.168 & -0.044 & 0.143 & -0.044 \\
\hline 7 & 2 & 1 & 4 & 8 & 0.172 & -0.122 & 0.186 & -0.001 & 0.174 & -0.055 & 0.170 & -0.052 & 0.147 & -0.052 \\
\hline 7 & 2 & 0 & 5 & 10 & 0.173 & -0.123 & 0.180 & -0.008 & 0.173 & -0.059 & 0.171 & -0.055 & 0.150 & -0.055 \\
\hline 8 & 2 & 2 & 4 & 8 & 0.151 & -0.101 & 0.178 & 0.019 & $\overline{0.156}$ & -0.035 & 0.148 & -0.032 & 0.126 & -0.032 \\
\hline 8 & 2 & 1 & 5 & 10 & 0.150 & -0.103 & 0.158 & 0.010 & 0.150 & -0.040 & 0.147 & -0.037 & 0.126 & -0.037 \\
\hline 8 & 2 & 0 & 6 & 12 & 0.149 & -0.102 & 0.150 & 0.006 & 0.146 & -0.042 & 0.146 & -0.038 & 0.127 & -0.038 \\
\hline 9 & 2 & 2 & 5 & 10 & 0.131 & -0.080 & 0.169 & 0.032 & 0.145 & -0.018 & 0.132 & -0.015 & 0.110 & -0.015 \\
\hline 9 & 2 & 1 & 6 & 12 & 0.125 & -0.080 & 0.143 & 0.027 & 0.127 & -0.020 & 0.122 & -0.017 & 0.102 & -0.017 \\
\hline 10 & 2 & 2 & 6 & 12 & 0.123 & -0.056 & 0.166 & -0.051 & 0.141 & 0.004 & 0.126 & 0.007 & 0.106 & 0.007 \\
\hline
\end{tabular}

$t_{S}^{L, \alpha}(\mathbf{r})$ in Eq. (12) for each functional. For the TF functional, the relative errors get smaller when the number of fermions increases. Showing much smaller absolute errors (probably due to a compensation of the TF errors by the opposite trend of the $\mathrm{vW}$ functional: its errors grow with the number of fermions ${ }^{12}$ ), the $\mathrm{TF} \frac{1}{5} \mathrm{vW}$ functional yields a similar behavior, except that the errors slightly increase for $N>8$. The GEA2 and both HCD functionals present no clear trends, because they appear to work better for those $N$-fermion configurations that are close to the closed-shell. And for all the functionals but the $\mathrm{TF} \frac{1}{5} \mathrm{vW}$ functional the relative error is smaller for configurations with smaller values of $\sum_{i=1}^{N} \sqrt{l_{i}\left(l_{i}+1\right)}$. Moreover, note that the errors are much smaller for the $\mathrm{TF} \frac{1}{5} \mathrm{vW}$ functional than for the GEA2 one, an opposite trend that we found for light atoms.

As explained in Section IC, $\sigma_{\min }$ is the amount of approximate KED misplaced with respect to the distribution $t_{S}^{L, \alpha}(\mathbf{r})$ after minimizing with respect to the parameter $\alpha$ - see Eq. (13). The values of the quality factor of Table In show that $\sigma_{\text {min }}$ decreases with the number of fermions for the TF functional. Even the relative errors of the kinetic energy are much smaller than for the TF case, the $\mathrm{TF} \frac{1}{5} \mathrm{vW}$ functional yields values of $\sigma$ that are bigger than those obtained for the TF functional, following the general results shown by the GGA functionals. $\frac{3}{3}$ Again, the local compensation of the TF behavior and the opposite trend of the $\mathrm{vW}$ functional ${ }^{12}$ cause the result for the $\mathrm{TF} \frac{1}{5} \mathrm{vW}$ functional. These results for $\sigma_{m i n}$, and the previous mentioned relative errors, arise from 


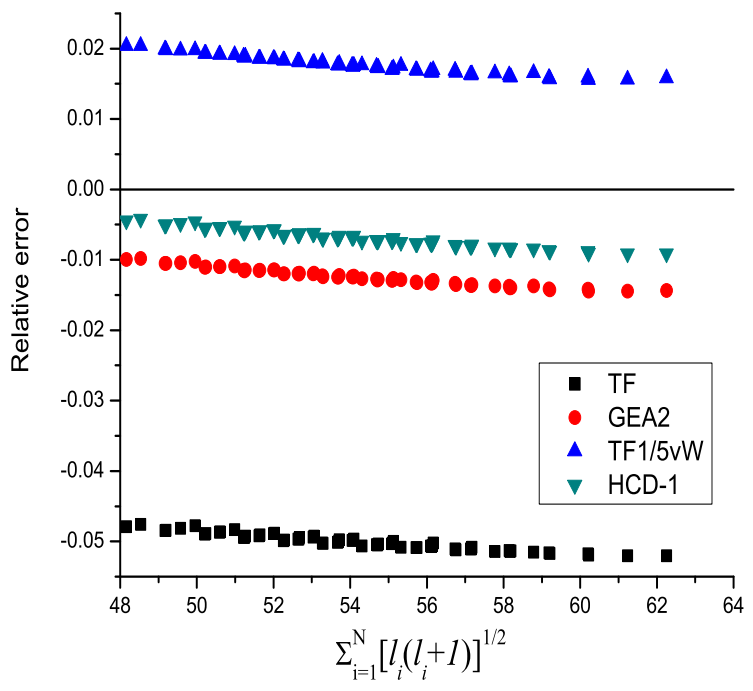

FIG. 3: (Color online) Relative errors obtained with the TF, GEA2, TF $\frac{1}{5} \mathrm{vW}$ and the first order correction HCD functionals in terms of $\sum_{i=1}^{N} \sqrt{l_{i}\left(l_{i}+1\right)}$, for all the 64 configurations of the confined noninteracting system with 34 fermions. Note that these results do not discriminate configurations with different values of $p_{4 s}$, as shown in the results for $\sigma$ of Fig.(4)

the known properties of the $\mathrm{vW}$ functional: it is a lower bound to both the total KE and the local KED. On the other hand, the general trend for the GEA2 and both first-order HCD functionals indicates that the quality factor decreases with the number of fermions, with quite similar results for the three approximations.

Focusing on the dependence on the angular momentum $\sum_{i=1}^{N} \sqrt{l_{i}\left(l_{i}+1\right)}$ of the $N$-fermion configurations we see that the local description of the TF functional improves for smaller angular momentum (i. e. the quality factors decrease with the angular momentum). The $\mathrm{TF} \frac{1}{5} \mathrm{vW}$ functional shows the opposite trend. On the other hand, the values of $\sigma$ for the GEA2 and the two HCD functionals decrease with the angular momentum when the shell is less than half-filled and increases when it is more than half-filled.

In order to make some other comparisons on the dependence on the angular momentum, let us discuss the case with $N=34$ fermions, that has 64 different configurations, all degenerate with the same energy. As commented before, these degenerate $N$-fermion systems have spherically symmetric densities, but due to the different occupancy of the $l$ single-fermion states they give different results for the kinetic energies and for the values of $\sigma$. Now we will focus on how the results obtained with each of the different KEDFs depend on $\sum_{i=1}^{N} \sqrt{l_{i}\left(l_{i}+1\right)}$. Regarding the relative errors, the HCD $r$-dependent functionals obviously give the same value since, once integrated, both yield the same kinetic energy. Fig. 3

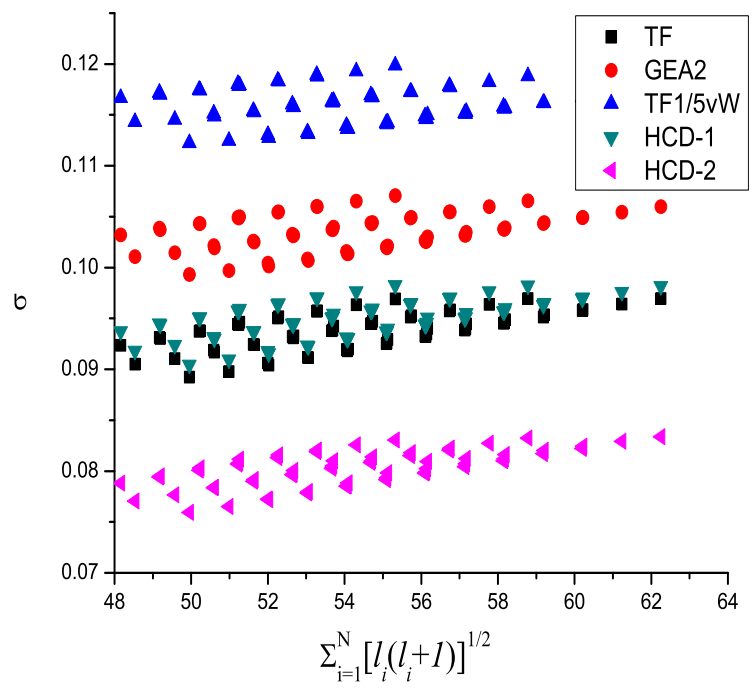

FIG. 4: (Color online) Quality factor $\sigma$ obtained with the $\mathrm{TF}, \mathrm{GEA} 2, \mathrm{TF} \frac{1}{5} \mathrm{vW}$ and the first order correction HCD functionals in terms of $\sum_{i=1}^{N} \sqrt{l_{i}\left(l_{i}+1\right)}$, for the 64 configurations of the noninteracting 34 -fermion system. Note the different results the two HCD functionals yield.

shows the values of the relative errors for the functionals for the noninteracting fermion system, as a function of $\sum_{i=1}^{N} \sqrt{l_{i}\left(l_{i}+1\right)}$. In this case, the HCD $r$-dependent functional gives the best results, better than the GEA2 ones, whereas the $\mathrm{TF} \frac{1}{5} \mathrm{vW}$ functional improves the values obtained with the TF one, getting again positive values. Note that for this system all the values of the relative errors are much smaller than for the atomic system.

Fig. 4 depicts $\sigma$ as a function of $\sum_{i=1}^{N} \sqrt{l_{i}\left(l_{i}+1\right)}$. In this case, the TF and HCD-1 functionals give very close results for $\sigma$, whereas the GEA2 functional yields worse values than the $\mathrm{TF}$ and the results of the $\mathrm{TF} \frac{1}{5} \mathrm{vW}$ functional clearly go bigger. To better understand the results, let us analyze how the fermions distribute among all the single-fermion orbitals. For $N=34$, the fermions occupy the lowest-energy orbitals, with six fermions in the fourth shell. Now we put our attention on the configurations that have a fixed value of $\sum_{i=1}^{N} \sqrt{l_{i}\left(l_{i}+1\right)}$, i. e. those results with the same value for the abscissas in the figure. Using the arguments presented in Ref. (12), for a fixed value of $\sum_{i=1}^{N} \sqrt{l_{i}\left(l_{i}+1\right)}$ the configurations with $n_{4 s}=2$ have more high- $l_{i}$ single-fermion orbitals occupied than the configurations with $n_{4 s}=1$; and when $n_{4 s}=0$ we will have our six fermions occupying orbitals with the lowest- $l_{i}$ possible values. Fig. 4 then shows that the values of $\sigma$ for the distinct configurations are clearly ordered in terms of the different values of $p_{4 s}$. This is not the case for the relative error of the energies, as can be seen in Fig. 3] where no differences are observed. This 
effect was also previously studied in Ref. (12).

\section{CONCLUSIONS}

The first-order correction HCD functionals improve the semilocal functionals (GEA2 and $T F \frac{1}{5} \mathrm{vW}$ ) we have used as benchmarks in the test systems we have studied. The relative errors are quite similar to the GEA2 functional for atoms, whereas they are slightly smaller for the noninteracting confined fermion system. We get accurate total kinetic energies, but when the local KEDs behavior is measured through $\sigma$, only the HCD-2 functional clearly gives better values than the TF functional, whereas the HCD-1 functional gets quite close results to those of the $\mathrm{TF}$ one. These results have revealed the differences in the local behavior of both HCD functionals. On the other hand, Figs. 2 and 4 show that the values of $\sigma$ are clearly smaller for the noninteracting fermion system than for the atomic system, reflecting the importance of the interaction on the KED.

As was also found in the previous paper, $\frac{12}{2}$ we have shown that for a specific number of fermions the results for the KEDs generally are different for each of the degenerate states of the noninteracting $N$-fermion system with spherical symmetric densities. Depicting their val- ues of $\sigma$ versus the values of $\sum_{i=1}^{N} \sqrt{l_{i}\left(l_{i}+1\right)}$ of each specific configuration some trends appear: for a given $\sum_{i=1}^{N} \sqrt{l_{i}\left(l_{i}+1\right)}$, those configurations whose occupied orbitals have the lowest values of $l_{i}$ compatible this value of $\sum_{i=1}^{N} \sqrt{l_{i}\left(l_{i}+1\right)}$ yield the smaller values for $\sigma$. As a consequence, the KEDFs discussed in the paper give a better description of the KED when the lower- $l_{i}$ singlefermion orbitals have the maximum occupancy. These KEDFs give a better description of the KED when the lower- $l_{i}$ single-fermion orbitals have the maximum occupancy.

An open question is to understand the reasons why one of these first-order correction HCD functionals gives better local behavior than any other KEDF in the literature.

\section{Acknowledgments}

Prof. R. G. Parr (University of North Carolina at Chapel Hill, USA) is grateful acknowledged by suggesting the study of the First-Order Gradient Correction to the Thomas-Fermi Kinetic Energy Functional that we present in this paper. This work has been partially supported by a grant of the Ministerio de Educación y Ciencia of Spain (reference FIS2007-65702-C02-02).
* Electronic address: dgaldea@fisfun.uned.es

$\dagger$ Electronic address: jealvar@fisfun.uned.es

¥ Electronic address: teresa.martin@upm.es

1 P. Hohenberg and W. Kohn, Phys. Rev. B 136, 864 (1964).

${ }^{2}$ W. Kohn and L. J. Sham, Phys. Rev. A 140, 1133 (1965).

3 D. García-Aldea and J. E. Alvarellos, J. Chem. Phys. 127, 144109 (2007).

4 S. Haq, P. K. Chattaraj, and B. M. Deb, Chem. Phys. Lett. 81, 8028 (1984).

5 B. M. Deb and P. K. Chattaraj, Phys. Rev. A 37, 4030 (1988).

6 P. K. Chattaraj, Phys Rev A. 41, 6505 (1990).

7 B. M. Deb and P. K. Chattaraj, Phys. Rev. A 45, 1412 (1992).

8 Z. Zhou, P. K. Chattaraj, R. G. Parr, and C. Lee, Theor. Chim. Acta 84, 237 (1992).

9 D. García-Aldea and J. E. Alvarellos, Phys. Rev. A 76, 052504 (2007).

10 D. García-Aldea and J. E. Alvarellos, Phys. Rev. A 77, 022502 (2008).

11 D. García-Aldea and J. E. Alvarellos, J. Chem. Phys. 129, 074103 (2008).

12 T. Martín-Blas, D. García-Aldea, and J. E. Alvarellos, J. Chem. Phys. 130, 034101 (2009).

13 L. H. Thomas, Proc. Camb. Phil. Soc. 23, 542 (1927).

14 E. Fermi, Rend. Accad. Lincei 6, 602 (1927).

15 C. F. V. Weizsacker, Z. Physik 96, 431 (1935).

16 D. A. Kirzhnits, Sov. Phys.- JETP 5, 64 (1957).

17 K. Yonei and Y. Tomishima, J. Phys. Soc. Jpn. 20, 1051 (1965).

18 Y. Tomishima and K. Yonei, J. Phys. Soc. Jpn. 21, 142
(1966).

19 S. R. Gadre and R. K. Pathak, Phys. Rev. A 25, 668 (1982).

20 R. A. Fisher, Proc. Cambridge Philos. Soc. 22, 700 (1925).

21 S. B. Sears, R. G. Parr, and V. Dinur, Isr. J. Chem. 19, 165 (1980).

22 S. Liu, J. Chem. Phys. 126, 191107 (2007).

${ }^{23}$ E. Romera and J. S. Dehesa, Phys. Rev. A 50, 256 (1994).

24 Z.-Z. Yang, S. Liu, and Y. A. Wang, Chem. Phys. Lett. 258, 30 (1996).

25 P. W. Atkins, Molecular Quantum Mechanics (Oxford University Press, 3rd. ed., Oxford, 1997).

26 E. Clementi and D. L. Raimondi, J. Chem. Phys. 38, 2686 (1963).

27 E. H. Lieb, Int. J. Quantum Chem. 24, 243 (1983).

28 M. Levy, Phys. Rev. A 26, 1200 (1982).

${ }^{29}$ H. Englisch and R. Englisch, Physica A 121, 253 (1983).

30 C. A. Ulrich and W. Kohn, Phys. Rev. Lett. 87, 093001 (2000).

31 Á. Nagy, Phys. Rev. A 57, 1672 (1998).

32 W. Yang, Y. Zhang, and P. W. Ayers, Phys. Rev. Lett. 84, 5172 (2000).

33 Á. Nagy, S. Liu, and L. Bartolloti, J. Chem. Phys. 122, 134107 (2005). 